\title{
Magneto-Inductive NEtworked Rescue System (MINERS): Taking Sensor Networks Underground
}

\author{
Andrew Markham and Niki Trigoni \\ Institute for the Future of Computing, Department of Computer Science \\ University of Oxford \\ firstname.lastname@cs.ox.ac.uk
}

\begin{abstract}
Wireless underground networks are an emerging technology which have application in a number of scenarios. For example, in a mining disaster, flooding or a collapse can isolate portions of underground tunnels, severing wired communication links and preventing radio communication. In this paper, we explore the use of low frequency magnetic fields for communication, and present a new hardware platform that features triaxial transmitter/receiver antenna loops. We point out that the fundamental problem of the magnetic channel is the limited bitrate at long ranges, due to the extreme path loss of $60 \mathrm{~dB} /$ decade. To this end, we present two complementary techniques to address this limitation. Firstly, we demonstrate magnetic vector modulation, a technique which modulates the three dimensional orientation of the magnetic vector. This increases the gross bitrate by a factor of over 2.5, without an increase in transmission power or bandwidth. Secondly, we show how in a multi-hop network latencies can be dramatically reduced by receiving multiple parallel streams of frequency multiplexed data in a many-to-one configuration. These techniques are demonstrated on a working hardware platform, which for flexible operation, features a software defined magnetic transceiver. Typical communication range is approximately $30 \mathrm{~m}$ through rock.
\end{abstract}

\section{Categories and Subject Descriptors}

C.2.1 [Computer-Communication Networks]: Network Architecture and Design

\section{General Terms}

Design, Experimentation, Measurement

\section{Keywords}

Search and Rescue, Magneto-Inductive, Magnetic, Mining, Triaxial, Network, Underground

Permission to make digital or hard copies of all or part of this work for personal or classroom use is granted without fee provided that copies are not made or distributed for profit or commercial advantage and that copies bear this notice and the full citation on the first page. To copy otherwise, to republish, to post on servers or to redistribute to lists, requires prior specific permission and/or a fee.

IPSN'12, April 16-20, 2012, Beijing, China

Copyright 2012 ACM 978-1-4503-1227-1/12/04 ...\$10.00.

\section{INTRODUCTION}

After a decade of intensive research in wireless sensor networks we are experiencing a flurry of sensing applications that rely on radio-based communication over the air. In stark contrast, sensor networks for underground environments are still in their infancy, and applications that could benefit from them are severely challenged. For example, in a mining disaster, a rockburst or collapse can isolate portions of underground tunnels, severing wired communication links and preventing radio communication. Miners who are trapped deep underground are unable to communicate with the surface.

In this paper, we introduce a communication layer based on low frequency magnetic communication. Operating in the near-field region, magnetic fields can penetrate rock, soil, fresh water and air with minimal attenuation. Due to the very low frequencies used $(2500 \mathrm{~Hz})$ wavelengths are in the order of kilometres and thus there is no multipath, another advantage over higher frequency radio.

However, unlike prior work [16] using a single loop antenna, to make the network operation invariant to node placement and orientation, each node is equipped with a triaxial loop antenna (which is used both as a transmitter and receiver). One of the challenges faced by magnetoinductive links is the inverse cube decay in signal strength with distance. In order to extend the communication range, nodes transmit over a narrow bandwidth, which helps to increase the SNR at the receiver but results in a low bitrate e.g. 32 bps. To improve the per-link bitrate, a novel technique, magnetic vector modulation, is introduced which modulates the transmitted signal over all three transmitter antennas, thus generating a magnetic vector whose amplitude and three-dimensional orientation can be controlled. The receiver, using its triaxial antenna, maps the detected magnetic vector to a signal constellation to decode symbols. We show that for the same transmitter power and bandwidth the effective bit rate can be increased by a factor of 2.58 .

With regard to the network itself, the low bitrates of the magnetic channel result in large multihop latencies, especially if nodes have to contend for the medium. Although the transmitter bandwidth is small, a wider band receiver (e.g. $2 \mathrm{kHz}$ ) can be used to simultaneously decode multiple frequency separated transmissions. As this is the opposite of one-to-many (broadcasting), we term this many-to-one approach broadcatching. We demonstrate that this technique can significantly reduce the latency of tree-based data collection, especially for aggregation queries. Used together in 
a network, these two techniques can reduce latency by over 4.5 times.

In summary this paper makes contributions in the following areas:

- We propose and have built magnetic transceiver nodes for underground sensor networks, that are able to transmit data through rock and soil at higher data rates than previously. We show how these nodes can fit in a hybrid sensor network architecture.

- We introduce magnetic vector modulation, a technique which modulates the spatial magnetic vector, more than doubling the effective bit rate, and also providing rotational invariance to node placement.

- We show how broadcatching can be used to reduce latency in the severely bandlimited magnetic channel.

- We demonstrate magnetic vector modulation and broadcatching on real hardware.

In Sec. 2, we discuss challenges and requirements the application scenario, underground mining. Sec. 3 presents our system design, motivating our new hardware platform and discussing the low bit rates of the magnetic channel. Sec 4 presents one solution to this problem by modulating the direction of the magnetic vector. Sec. 5 introduces a complementary solution which increases the overall network throughput. The benefits of the two solutions are evaluated on real hardware and in simulation in Sec. 6. The contributions of the work are contrasted with existing literature in Sec. 7 and we summarize the contributions in Sec. 8

\section{MOTIVATING SCENARIO: MINING}

As a motivating example, we consider communication in an underground mine.

\subsection{Mine Operations and Disasters}

There are two broad classes of mining operations, surface and underground. Typically an underground mine will have a central shaft following the dip of the ore body and usually about $10 \mathrm{~m}$ below the ore body. From this will radiate access tunnels that are slightly angled from the horizontal to manage water drainage. There are larger tunnels, usually separated by a $30 \mathrm{~m}$ depth difference that are designed to transport ore using electric locomotives or conveyors. Mining is a hazardous occupation, with a fatality rate in 2007 of 21.4 per 100,000 fulltime workers in the United States [1]. Compared with the average rate for all private industry of 4.3 per 100,000 , the fatality rate is nearly five times as large [1]. Within the mining sector, underground mining is significantly more dangerous than surface operations, with a fatality rate about twice as high [5]. Dangers include rockbursts which generally lead to a collapse of a tunnel, fire and explosion, a major risk in coal operations, and flooding. The rescue of 33 trapped Chilean miners in 2010 after 69 days underground brought the importance of underground safety and communication to the forefront of global awareness. However, communication was only made possible after sinking shafts. Along with the successful rescues, there have also been terrible tragedies. The Sago Mine disaster on January 22006 in Virginia, USA [9] is a particularly relevant example. An underground explosion in a coal mine trapped twelve miners behind a blast curtain. Although the way out was clear, they had no way of knowing as the mine telephone system had been destroyed during the blast and by the time rescuers reached them, all but one had died from carbon monoxide poisoning. This tragic example highlights how underground communication systems have to be improved and made robust to roof collapse and flooding.

In the event of a mining disaster a communication system could provide information such as: the number of miners still underground; their approximate location; concentrations of gases (methane, oxygen, carbon dioxide); flooding and whether miners can move freely or are trapped. In addition, a two-way communication link, even if operating over low baud rates, can be used to send messages to trapped miners, such as which access shaft they should move to to facilate rescue operations.

\subsection{Mine Communication}

Percussion: Trapped miners can communicate with rescue teams by banging on rock bolts with metal pipes and sledge-hammers. Rescue teams can use sensitive geophones to listen for rhythmic banging and try and localize the miners. This method is obviously exhausting for the trapped miners and cannot be used for effective communication to tell miners how best to escape.

Mine Telephone and Wired links: Modern mines are fully wired, often with ethernet or optical links. This allows for high speed data transmission and monitoring. However, in a collapse, flood or fire, wired links can be broken, preventing communication with other sections of the mine. In addition, wired links cannot be used directly to communicate with miners and require a bridge to wireless technologies.

Leaky Feeder: This is a "semi-wireless" approach where a coaxial cable with periodic breaks in the shield capacitively couples with a receiver device, enabling short range wireless communication. Amplified repeaters are placed every few hundred metres in order to boost the signal.

Wireless: Researchers have attempted to provide a communications infrastructure underground by using off-theshelf technology such as Zigbee [4]. Due to the waveguide like nature of tunnels and sharp corners, high frequency radio waves suffer from severe multipath and heavy attenuation. Radio cannot be used to communicate through rock or soil in the event of a collapse, a fundamental drawback [18]. Through-the-earth: An alternative approach to transmitting information to miners is the use of low frequency magnetic fields, which are able to penetrate soil and rock strata largely unattenuated, so called through-the-earth (TTE) systems [20]. The most widely adopted system is the one-way PED (personal emergency device) system which consists of a very large loop antenna placed on the surface driven with over $1.5 \mathrm{~kW}$ of power. This allows it to penetrate a few hundred metres of rock, depending on the conductivity. Its drawback is that it is only a one-way communication link and does not allow miners to send messages to the surface. The reason for this is that a device which is small enough to be carried by a miner is unable to generate a large enough magnetic field to be detected at the surface.

There is a clear need for a communication system which is able to maintain two-way connectivity in the event of a mining disaster. In the next section we propose a new node design and a hybrid network architecture that can meet these 


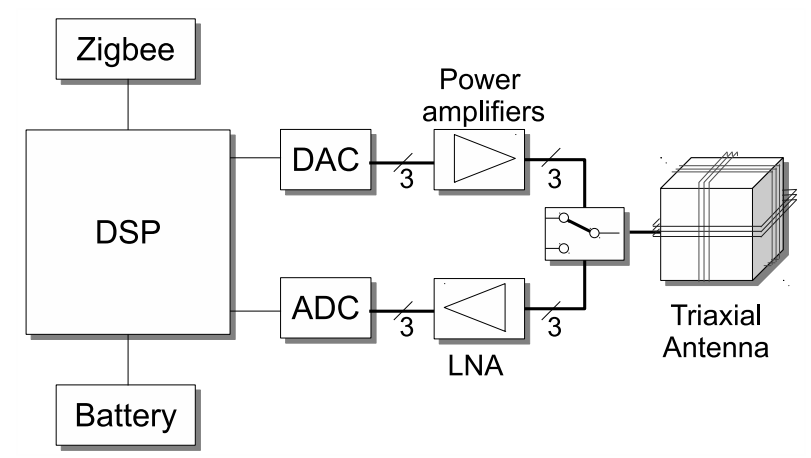

Figure 1: Block diagram of a magnetic transceiver node.

requirements. Note, however, that our proposed system is not applicable only to the mining scenario; it can be used in a wide range of scenarios that involve underground operations, such as geological studies, volcano monitoring, precision agriculture and animal monitoring.

\section{SYSTEM DESIGN}

The operational requirements as dictated by the application of miner rescue are:

- Information from trapped miners should be relayed to the surface with minimal latency

- The system should be able to maintain communication in the event of rockfall or failure of existing communication modalities

- The magnetic transceiver nodes should be small enough to be regularly deployed in an operational mine

- The nodes should not require precise placement or positioning

In this section, the design of our new node platform is presented and the design choices made at each level of the stack are highlighted. We then explain how our platform fits in a hybrid sensor network architecture that combines wired, radio and magnetic communication channels.

\subsection{Node Design}

The block diagram of a magnetic transceiver node is shown in Fig. 1. The digital signal processor (DSP) lies at the heart of the system and essentially acts as a software defined radio (SDR). The use of SDR allows for great flexibility in designing and evaluating different modulation schemes over the magnetic channel. Through analog-to-digital (ADC) and digital-to-analog (DAC) convertors the DSP modulates and receives data from the magnetic channel. The magnetic field is generated and detected by the triaxial antenna on the right hand side of the figure. The node is also equipped with a Zigbee (802.15.4) transceiver to allow for short range, high-data rate communication. We first discuss the design of the physical layer, namely how to generate a magnetic field, effects on the magnetic field due to the channel, and how to detect a magnetic field.

\subsubsection{Generating a magnetic field}

A current passing through a coil generates a magnetic field in the space around it. If the coil dimensions are small relative to the distance to the receiver, it can be approximated as a point dipole source, most conveniently expressed in spherical co-ordinates as

$$
\vec{B}=\frac{\mu_{0} \mu_{r} N I A}{4 \pi r^{3}}(2 \cos (\theta) \hat{r}+\sin (\theta) \hat{\theta})
$$

where $\mu_{0}=4 \pi 10^{-7} \mathrm{H} / \mathrm{m}$ is the permeability of free space, $\mu_{r}$ is the relative permeability of the medium, $N$ is the number of turns, $I$ is the current flowing through the coil, $A$ is the cross sectional area, $\theta$ is the azimuthal angle, $\hat{\theta}$ is the unit normal vector in the azimuthal direction, $\hat{r}$ is the unit radial vector and $r$ the distance between the source and the sensor [8]. There are a number of important factors to note about this equation which guide the design process.

Rolloff: The magnitude of the magnetic field decays with increasing range as an inverse cube $\left(\propto r^{-3}\right)$, rather than following an inverse square law as is typical for electromagnetic radiation in free space. This results in a $60 \mathrm{~dB} /$ decade attenuation in signal strength with range. This rolloff significantly reduces the range that can be practically obtained. For example, to double the range of a given transmitter, the magnetic moment has to be increased eightfold. Hence to span a given distance, it is more power efficient to use many smaller hops, rather than one single large hop. This factor suggests the use of distributed multi-hop networks [15].

Coupling: Soil, air, water and most rocks have a relative permeability $\mu_{r}$ very close to 1 . This means that magnetic fields are not attenuated differently by these media and hence can be used for communication through rock. However, ferrous materials such as iron, steel and nickel have much higher permeabilities, ranging between 100 and 600, depending on composition. Rather than preventing communication, induced eddy currents in the conductor can actually be used to increase transmission range, with the metal object acting as a passive re-radiator [20].

\subsubsection{Magnetic Channel}

With respect to the magnetic channel, there are two effects to consider, namely frequency dependent absorption (skin effect) and background noise. These factors dictate the operational frequency that the magnetic transceiver node adopts.

Skin effect: The conductivity of rock and other objects has a frequency dependent effect on the magnetic field. This is because a time-varying field induces a current in the rock, which acts to cancel the magnetic field. Thus, even though the media can have a permeability close to 1 , over long distances the skin effect starts to dominate the channel. The skin depth, the distance at which a signal is attenuated to $1 / e=0.368$, is given as

$$
\delta=\sqrt{\frac{2}{\omega \mu_{0} \sigma}}
$$

where $\omega$ is the frequency and $\sigma$ is the conductivity of the media, measured in mho/m [7]. For a typical overburden conductivity of $0.01 \mathrm{mho} / \mathrm{m}$, the skin depth at $5 \mathrm{kHz}$ is $71 \mathrm{~m}$

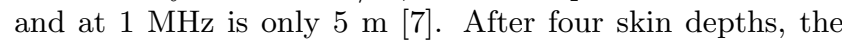
signal is attenuated by over $20 \mathrm{~dB}$. Eq. 2 demonstrates that the use of higher carrier frequencies comes at the cost of significantly increased attenuation. 


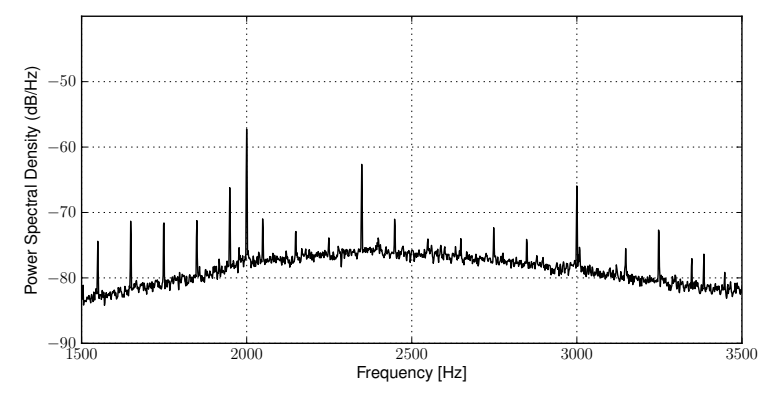

(a)

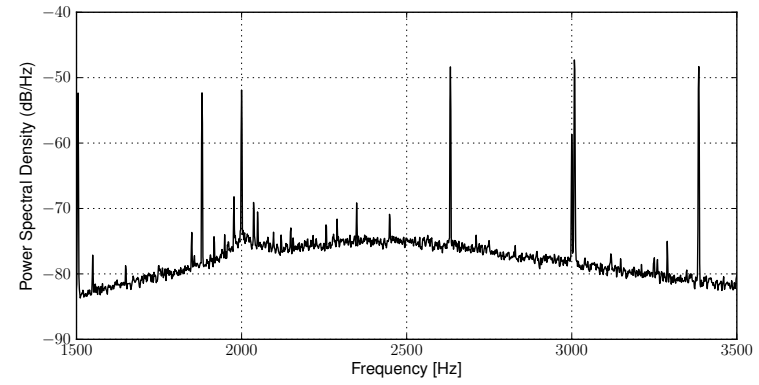

(b)

Figure 2: Power spectral density of background noise. (a) shows the PSD as captured by the x-axis sensor. (b) shows the PSD as captured by the z-axis sensor.

Background noise: The magnetic channel is dominated by low frequency noise, caused by a variety of natural and man-made factors, such as lightning, mains noise and earth resonance. Noise can be approximated as roughly following a $1 / f$ distribution, with strong narrowband interferers.

The power spectral density (PSD) of background noise in the range of $1500-3000 \mathrm{~Hz}$ is shown in Fig. 2 for the $\mathrm{x}$ and $\mathrm{z}$ channels. The mains harmonics can be seen as strong peaks, as high as $30 \mathrm{~dB}$ above the noise floor, in the PSD plot. Note also that the harmonics are different for the $\mathrm{x}$ and $\mathrm{z}$ channels as the noise sources are directional. To avoid the strong interference, channels can be centred halfway between adjacent harmonics.

Together, these competing constraints dictate operation around the very low frequency (VLF) frequency band $(3 \mathrm{kHz}$ to $30 \mathrm{kHz}$ ). At these low frequencies, the wavelengths are typically much larger than the distance between nodes, ranging from $100 \mathrm{~km}$ to $3 \mathrm{~km}$ respectively. Operation is thus in the reactive (quasi-static) near field. The field is nonpropagating and hence there can be no multipath or fading.

\subsubsection{Detecting a Magnetic Field}

There are a number of sensors which can be used to detect magnetic fields, such as solid state magnetometers, SQUIDS and search coils. All of these are based on changes in measurable characteristics, such as resistance or voltage which are created in response to an incident magnetic field. For simplicity, the same transmitting coil is used as a receiving loop. The voltage produced by a multi-turn coil is

$$
V=\mu_{0} N A 2 \pi f B \cos \theta,
$$

where $N$ is the number of turns, $A$ the cross-sectional area $f$ the carrier frequency, $\vec{B}$ the magnetic field vector and $\theta$ is the angle between the magnetic field and sensor.

Angular dependence: The $\cos \theta$ term in Eq. 3 (essentially the dot product between the magnetic field vector and the unit normal to the antenna aperture) has a very significant effect on reducing the robustness of the communication channel. As $\theta \rightarrow \frac{\pi}{2}$, the term $\cos \theta \rightarrow 0$. Essentially, when source and sensor are mutually perpendicular, the sensor cuts no lines of flux and hence communication is impossible.

If magnetic nodes are equipped with single loops, as in [16], deployments have to be carefully made such that nodes line up along a common axis. This is not robust enough for many applications, in particular mining, and thus a solution using three mutually orthogonal receiver coils is used. With this configuration, as the axes of the antenna are at right angles to each other, as one antenna's signal is minimized, another is maximized. Thus, the total received voltage is never zero. Note that there is still an amplitude dependence due to the impact of the radial component of the magnetic field being twice as high as the tangential component in Eq. 1. However, if the transmitter is also equipped with triaxial antennas, and the signal to be transmitted is whitened such that no one antenna is favored, then the received power at various orientations is constant. Note that a triaxial coil arrangement permits operation with arbitrarily positioned and oriented nodes in 3-D space, but if only a planar (2D) layout is required, nodes can be equipped with biaxial orthogonal coils.

\subsubsection{Summary of Design Choices}

Motivated by the directional nature of uniaxial magnetic loops, each transceiver node is equipped with three orthogonal antennas, making the system rotationally invariant. However, due to the narrow channel bandwidth, the achievable bit rates are very low, e.g. 32 bps. By adopting the triaxial antenna configuration, both on transmitter and receiver, we demonstrate in Sec. 4 that by modulating the orientation of the magnetic vector, more information can be encoded on the channel per unit of energy. This increases the effective bit rate.

\subsection{Network Design}

In this section, we consider how a network comprised of magnetic transceivers would operate, under the constraints highlighted in Sec. 3.1. This is shown graphically in Fig. 3. Each transceiver is equipped with wired and wireless (RF) links which are used in normal mining operations, transferring information from workers and sensors about position and conditions. Sensors can either be wired or wireless and data is forwarded to each magnetic transceiver node which then relays the information over a wired or wireless link to another magnetic transceiver, until it reaches the surface. Control messages can also be relayed from the surface to actuate various underground machinery. A hybrid wired/wireless approach is used as the limited bitrate of the magnetic channel is not adequate for normal operations. Miners are equipped with small Zigbee based transceivers, with various sensors such as accelerometers. These emit pe- 


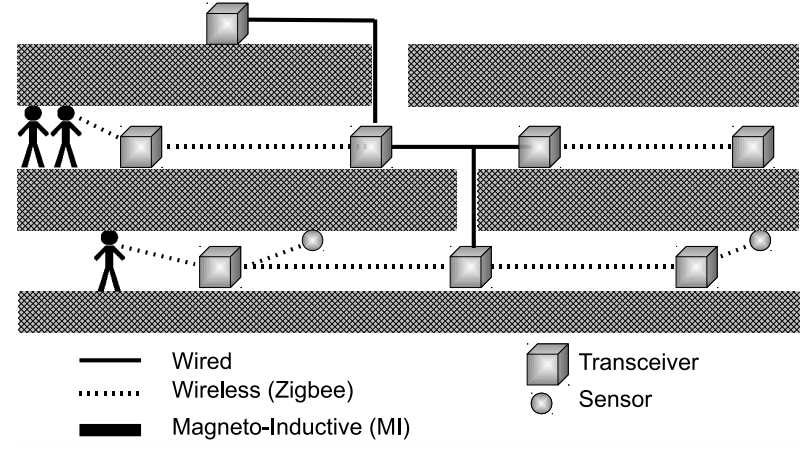

(a)

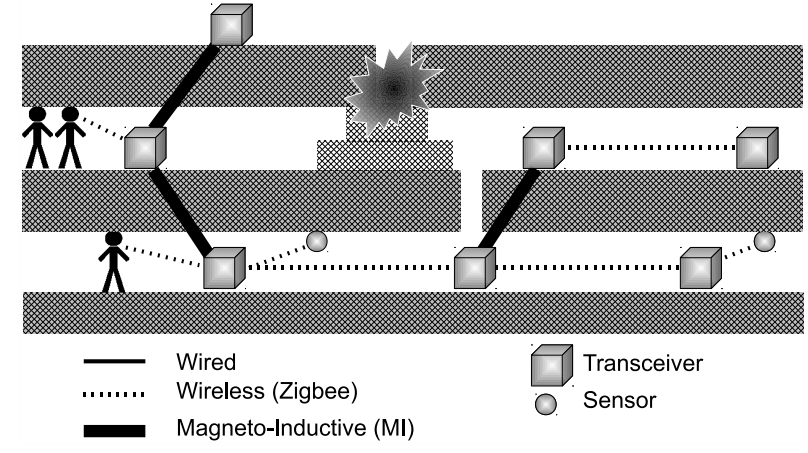

(b)

Figure 3: Network overview. (a) Shows normal operation. The mine-wide sensing system communicates through a combination of wired and wireless links, transferring information from sensors and people to the surface. (b) Shows operation in a disaster scenario. The network re-organizes and uses low frequency magneto-inductive communication to bridge the destroyed links, penetrating rock. Thus the positions of the trapped miners can be relayed to the surface.

riodic, uniquely coded radio beacons which are detected by the magnetic transceivers when they are within range or via multi-hop data gathering.

In the event of a disaster, which can break wired communication and prevent wireless communication, the system falls over to low-bandwidth magneto-inductive communication, which is able to penetrate rock and soil, maintaining network connectivity. The magnetic transceiver nodes are equipped with backup batteries to enable operation to continue even if power fails. The magnetic transceiver nodes can also be equipped with sensors themselves to directly measure parameters such as carbon monoxide concentration or temperature. They can also query wireless sensor devices and hence act as a gateway between the surface and underground. Thus, this system aims to augment existing wireless technology by providing a robust backup path.

The low bitrate magnetic channel has significant implications for network operation, in particular the latency of query responses. To this end, we present two complementary techniques to dramatically speed up data dissemination through a magnetic network: 1) magnetic vector modulation, a technique at the physical layer discussed in Sec. 4), and 2) broadcatching, a technique at the medium access control layer discussed in Sec. 5 .

\section{MAGNETIC VECTOR MODULATION}

The use of tri-axial sensors to measure vector fields is commonplace, e.g. tri-axial accelerometers. What is less well known is that tri-axial transmitters (comprising three mutually orthogonal loops) can be used to generate magnetic vector fields. By controlling the phases and amplitudes of the currents in each transmitting coil, the three dimensional vector field at a receiver can be oriented in any arbitrary direction. In this way, more information can be encoded using the same available bandwidth and the same total transmitter power.

A simple demonstration of the principles behind magnetic vector modulation is shown in Fig. 4. At each time step, a separate coil is energized. The magnetic fields detected by the sensors at the three different locations A, B and C vary in magnitude and polarity depending on which coil is energized. Thus it can be seen that by changing the threedimensional current phasing of the source, the magnetic field vector detected at the receiver also changes. This provides the basis for the communication system.

Transmitter Chain: In scalar BPSK, a constant frequency carrier is multiplied by a lower frequency bipolar data message, with values of +1 and -1 . Scalar BPSK results in a maximum theoretical spectral efficiency of $1 \mathrm{bit} / \mathrm{s} / \mathrm{Hz}$. There are many possible ways to convert BPSK to a vector based representation. For simplicity, we allow a single coil to be energized per symbol (either with positive or negative phase) and the other two coils are left open. This gives a total of $M=6$ possible symbol values. These are shown in Table 1, along with the corresponding baseband currents in each coil.

To send a data message, the transmitter first sends a carrier tone, followed by a multichannel preample. This allows the receiver to learn the channel transfer function. Following this, the incoming serial binary data stream is mapped to a base- 6 representation. The symbol mapping dictates which coils are energized at a particular time and their respective phasing. The baseband signals are then modulated with the higher frequency carrier wave and after amplification, applied to the transmitter coils. To reduces the overall bandwidth of the signal it is passed through a root raised cosine pulse shaping filter.

Table 1: Coil energizations

\begin{tabular}{|c|c|c|c|}
\hline Symbol & $i_{x}$ & $i_{y}$ & $i_{z}$ \\
\hline 0 & +1 & 0 & 0 \\
\hline 1 & 0 & +1 & 0 \\
\hline 2 & 0 & 0 & +1 \\
\hline 3 & -1 & 0 & 0 \\
\hline 4 & 0 & -1 & 0 \\
\hline 5 & 0 & 0 & -1 \\
\hline
\end{tabular}




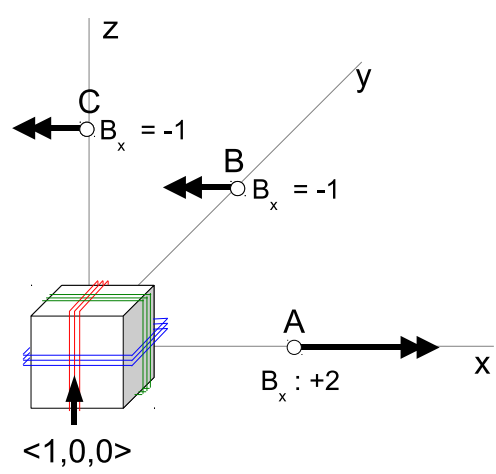

(a)

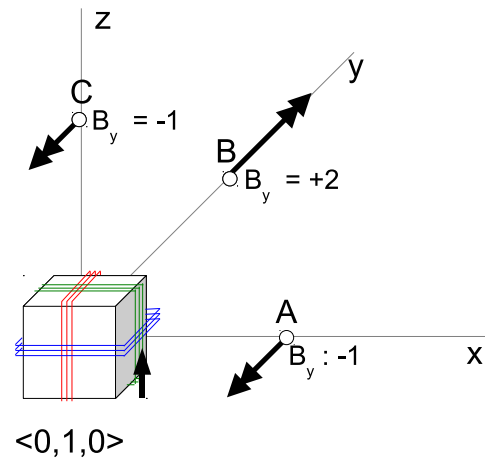

(b)

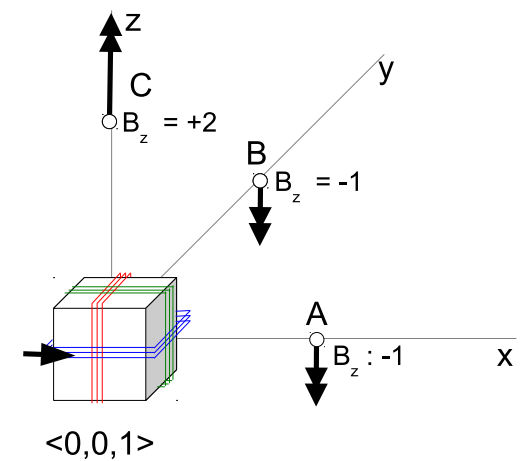

(c)

Figure 4: The basic method of communication: a triaxial source of magnetic field, shown as a cube at the origin, generates magnetic fields. The resultant magnetic fields at three different sensors placed on the $\mathrm{x}$-axis (A), the $y$-axis (B) and the z-axis (C) are shown. (a) The $X$ coil in the transmitter is energized. Note the field at $A$ is twice as large and opposite in sign to the fields at $B$ and $C$. (b) The $Y$ coil in the transmitter is energized. (c) The $\mathrm{Z}$ coil in the transmitter is energized.

Receiver chain: The receiver chain is shown in Fig. 5. Like a conventional receiver, the weak signals are first amplified by a low noise amplifier followed by automatic gain control to maintain an acceptable dynamic range. The three channels are downconverted using a complex carrier to form in-phase (real) and quadrature (imaginary) components.

Similar to a normal single channel receiver, the first block in the chain is carrier phase/frequency tracking to adjust the local oscillator's generated frequency in order to maintain phase synchronicity. This is performed using a Costas Locked Loop which synthesizes a single carrier, not three separate ones. This is because there is frequency or phase shift between the three channels. The error signal applied to the feedback loop is derived from all three input channels, giving more weight to those with better signal-to-noise ratios. The next stage in the receiver chain is to perform timing recovery. This is like a normal receiver except that the vector amplitude (i.e. the RMS amplitude of the three channels) of the incoming signal is used to control the data sampler, which determines at which point a symbol sample should be taken to maximize the energy.

The following stage in the process is the block used to estimate the channel transfer function, $\mathbf{H}$. This is a $3 \times 3$ matrix that contains coefficients which represent the coupling between the transmitter and receiver antennas. Note that although there are six symbols, only three vector coefficients need to be stored as the remaining three are simply opposite in sign and lie in an antipodal position within the constellation. The transmitter sends a known preamble character on each of the three channels in sequence. The receiver averages the 3 -D magnetic vector it receives over the duration of each preamble character. Each of these is used to populate a column of $\mathbf{H}$. Note that the magnetic vector is purely real, as no phase shift can occur due to multipath. As shown in Fig. 4, the $\mathbf{H}$ matrix will have different values depending on the relative separation and orientation between the transmitter and receiver.

To decode the incoming data, the receiver chooses the symbol that minimizes the Euclidean distance between the

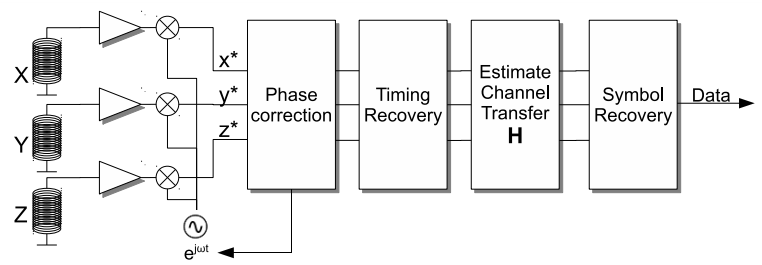

Figure 5: System diagram showing the receiver communication chain.

received 3-D vector and the known $\mathbf{H}$. The 6-ary symbols are then unmapped to recover the serial data stream. This is explained with reference to an example constellation shown in Fig. 6. The constellation is established using $\mathbf{H}$ determined during the initial channel estimation phase. The preamble symbols are averaged to form a cluster, where the centroid of the cluster is the respective column vector in $\mathbf{H}$. To decode a symbol, the 3-D Euclidean distance between the received vector signal measured from all three channels, marked with an ' $\mathrm{x}$ ' in the figure, is determined to every point in the symbol constellation. A hard decision is made based on which point of the constellation the symbol is closest to, returning in this case, the symbol 0 .

To compare against the scalar BPSK case, note that the power consumed is identical, as only one coil is energized at each point in time. Also, note that the bandwidth occupied is identical. However, instead of sending $M=2$ possible symbol values, in the vector case $M=6$ symbol values have been sent. Thus, the gross bitrate has been increased by a factor $\frac{\log _{2}(6)}{\log _{2}(2)}=2.58$.

Note that compared to the scalar BPSK case, an extra two preamble characters have to be sent. Assume that the length of each preamble character is $\mathrm{P}$ symbols, and that scalar BPSK also sends a preamble byte to synchronize the 


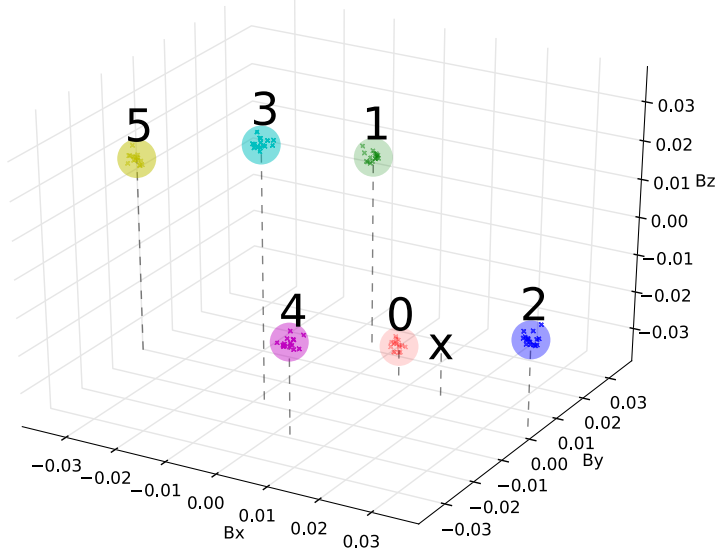

Figure 6: Example constellation showing how the incoming symbol, marked by ' $x$ ' is decoded. It is closest within the three-dimensional magnetic field strength to symbol 0.

receiver, then the time taken to send $\mathrm{D}$ bits of data is

$$
t_{\text {scalar }}=t_{s}(P+D),
$$

where $t_{s}$ is the symbol time. For the vector case, the total time taken to send D bits of data is

$$
t_{\text {vector }}=t_{s}\left(3 P+\frac{D}{\log _{2}(6)}\right) .
$$

For a preamble character length of $P=8$ bits, magnetic vector modulation is more efficient if the data is longer than $D=32$ bits or 4 bytes. Thus, it can be seen that the additional overhead of determining the channel transfer function is negligible for the magnetic vector modulation case.

Note that any modulation schemes can be extended using these extra orthogonal dimensions. For example, a transmitter can use quadrature modulation by using a complex carrier. The drawback of the magnetic vector approach is that the transmitter and receiver chains needs to be triplicated, which can increase cost and complexity. However, the advantages such as rotational invariance and increased bit rate far outweigh the additional hardware and software requirements.

\section{BROADCATCHING}

The small transmitter bandwidth of the transmitter in Sec. 3 was highlighted as a potential drawback of the magnetic channel. Magnetic vector modulation (Sec. 4) was presented as one solution to this problem that does not consume additional energy or bandwidth. The small transmitter bandwidth has the effect of limiting the maximum achievable bit rate. For example, within a bandwidth of 32 $\mathrm{Hz}$, the bit rate, even when using magnetic vector modulation is approximately $80 \mathrm{bps}$. Although the transmitter bandwidth is very small $(32 \mathrm{~Hz})$, the receiver is able to capture signals over a wider range, e.g. $2000 \mathrm{~Hz}$. Furthermore, due to the low operating frequencies and the software defined demodulator, it is computationally simple and feasible to simultaneously decode multiple, frequency separated, data streams. This is similar to orthogonal frequency division multiple access (OFDMA) which is used in multichannel
WiFi [21]. We term this many-to-one concept broadcatching, as it is the reverse of broadcasting, which is one-to-many. Broadcatching has some significant implications for the low data rate, high latency magnetic network.

Contention free: The first implication of broadcatching is that through frequency division, contention can be eliminated entirely (static channel allocation through graph coloring [6]) or greatly reduced (random channel allocation). This is important as the high latencies of the magnetic channel will be exacerbated if nodes have to contend for access to the medium. Interference will also be eliminated, which again is important both in terms of latency and energy consumption, as failed packets have to be retransmitted and hence waste energy and bandwidth. In the prototype system, if channels are spaced every $50 \mathrm{~Hz}, 40$ distinct frequency channels can be packed into a $2 \mathrm{kHz}$ bandwidth. Given the low probability of collision, nodes can randomly hop to a new channel for each transmission. Although this introduces contention, the probability of interference is very low. Also note that the receiver does not need to be aware of the channel hopping sequence and it can be completely random. In terms of implementation, the number of channel decoders does not need to be equal to the number of input channels, only to the number of children. Channel decoders only need to be executed when a valid signal is received. Thus, channel decoders can be dynamically mapped to particular frequency bands. Channels with low SNR, such as those with strong mains harmonics can be avoided.

Network Algorithms: The second major difference is in operation of standard network algorithms, such as tree collect and query propagation. In a mine rescue scenario, aggregation queries such as sum, max, average etc., can be used to determine the number of people underground, the maximum methane concentration, the minimum oxygen concentration and so forth. It is important for a rescue that such information can be gathered rapidly.

To execute an aggregation query, data originates at the leaves of the query tree and percolates upwards. Once each parent has obtained all the data from its children, it combines its own sensor value with the query function, ready to send to its parent. In conventional wireless sensor networks, this means that each child must send its data in a time-slotted fashion to its parent, in order to prevent collisions at the receiver. Hence, the time taken for each node to gather the data to execute the query is related to the number of children it has. In general, if each parent has $B$ children, and the depth of the tree is $D$, then the total time taken to execute the aggregation query using conventional time slots is proportional to $D B$. This implies that to minimize the time taken to execute the query, the breadth $B$ and the depth $D$ of the tree should be equal. In addition, trees should be balanced such that each node has an approximately equal number of children in order to minimize the maximum breadth.

In the broadcatching tree, the children can all send their data during the same time slot. Thus, the number of children that a node has does not alter the data gathering time. Thus, the total time taken to collect information from such a tree is proportional to $D$ units of time, where $D$ is the depth of the tree. This is an important result, as the execution time is independent on the number of nodes in the network and only related to the tree depth. It also suggests that trees should be broad rather than deep and that bal- 


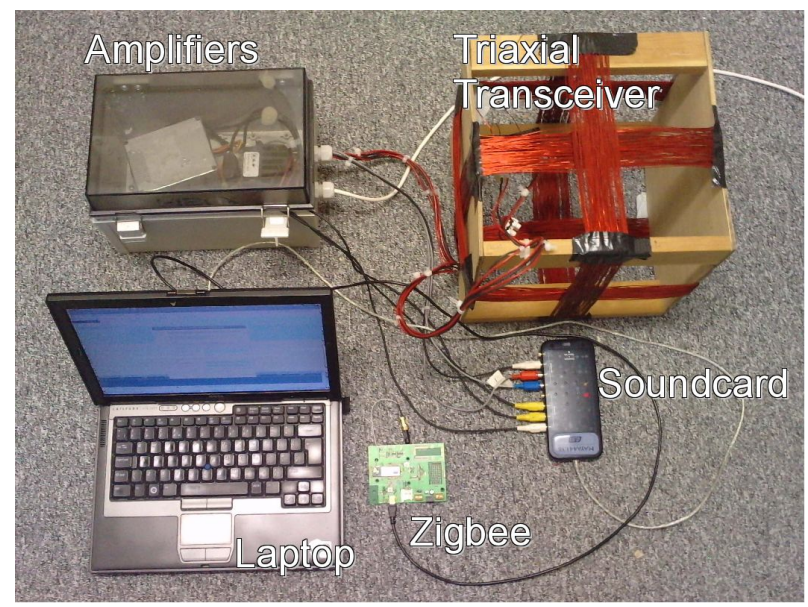

Figure 7: The proof-of-concept transceiver.

ancing the number of children per node is not important, subject to channel availability. It is also more energy efficient to use a broadcatching tree, as nodes do not need to stay awake to gather data over multiple time periods.

Broadcatching can also be used in decentralized algorithms which require state information to be gathered from all their one hop neighbours [6]. If nodes are divided into two groups, such that no nodes which share a link are in the same group, then nodes can establish a two stage cycle, alternating between transmitting and receiving. At the end of the cycle, all nodes will have complete state information from all their neighbours.

\section{EXPERIMENTAL RESULTS}

In order to demonstrate the principles of magnetic vector modulation, a small testbed was constructed. The key concept was to take advantage of software defined radio (SDR) principles to allow for maximum flexibility in testing different symbol rates, carrier frequencies and modulation schemes.

\subsection{Hardware implementation}

Magnetic Transceiver Node: A magnetic transceiver node is shown in Fig. 7. In the implementation, a laptop is used as a DSP, due to the low computational requirements and to enable rapid prototyping. The magnetic transceiver is interfaced through a 4 channel USB external soundcard (MAYA44-USB). The transmitted signals are amplified using conventional Class-B audio amplifiers. The antenna loops are equipped with series capacitors to enable them to resonate at the carrier frequency. The received signal for each loop is amplified using an LT1028 precision low power instrumentation amplifier, operating in transimpedance configuration (current feedback) to allow for a wide bandwidth to be obtained. The low noise amplifiers are placed inside a shielded metal box to minimize interference and noise. A Zigbee interface is also provided via USB. A C++ program was written to generate and decode the magnetic vector modulation signals. With the current implementation, the system is able to broadcatch up to 15 magnetic vector modulation streams simultaneously. The same coils were used for both transmitting and receiving, and each consisted of

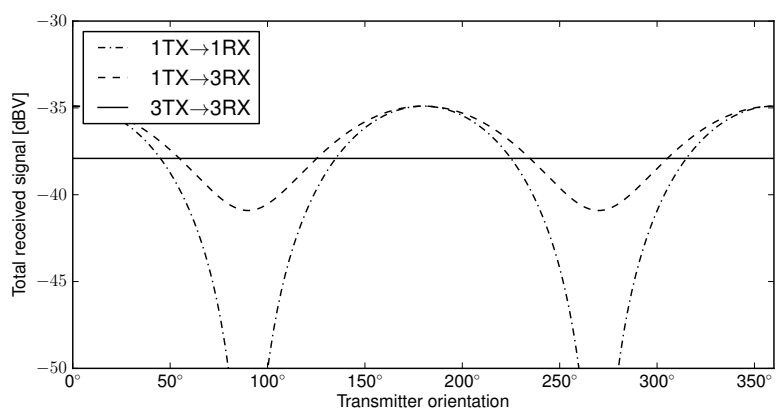

Figure 8: Impact on total received power with different combinations of receiver and transmitter antennas, as the transmitter is rotated.

80 turns of $0.5 \mathrm{~mm}$ diameter enamelled copper wire wound over a wooden cube with sides $30 \mathrm{~cm}$. In the following experiments, the effective magnetic moment of each loop is 7.2 $\mathrm{Am}^{2}$.

Miner unit: The miner unit is a miniature Atmel ATZBA2 Zigbit module, which contains an 8 bit Atmega1281V microcontroller and a Zigbee transceiver. It has also been equipped with a low power triaxial accelerometer, LIS302DL, and an LED which simulates a miner's cap lamp and which flashes when an evacuation message is received. To reduce bandwidth, the dynamic RMS acceleration is averaged over a 60 second moving average window. This data is periodically beaconed, along with the node's ID.

\subsection{Magnetic link}

Rotational invariance: The primary motivation for the adoption of triaxial transceivers in Sec. 3.1.3 was the fact that the relative orientation of the transmitter-receiver pair was irrelevant. This is shown in Fig. 8. Note how with a single antenna on both the transmitter and receiver, there are strong nulls at $90^{\circ}$ and $270^{\circ}$, preventing communication. If the receiver is equipped with a vector antenna, then regardless of the orientation, a signal can be obtained. There is still an amplitude variation, due to the factor 2 in the radial component of Eq. 1. If vector antennas are used on both transmitter and receiver, the signal received is constant, regardless of relative orientation, as shown by the solid line in Fig. 8. This approach makes deployment of a magnetoinductive wireless network simple, as devices do not need to be placed in a line or plane.

Vector modulation: An example of a magnetic vector modulated signal is shown in Fig. 9 for a transmitter/receiver separation of $5 \mathrm{~m}$. In Fig. 9(a), it can be seen that only one transmitter antenna is active at a time. The received signal in Fig. 9(b) shows how each received channel is a linear combination of the transmitter channels and varies in amplitude according to the active antenna. Note how when the $\mathrm{X}$-axis on the transmitter is energized, the strongest signal actually appears in the $\mathrm{Y}$-axis of the receiver. These values alter as the transmitter-receiver orientation and location is changed. The frame begins with a carrier tone, which enables the receiver to phase-lock to the transmitters relative phase and frequency. This is followed by a preamble on each channel in turn. The receiver uses this to compensate for the inherent $180^{\circ}$ phase ambiguity presented by the use of BPSK. 


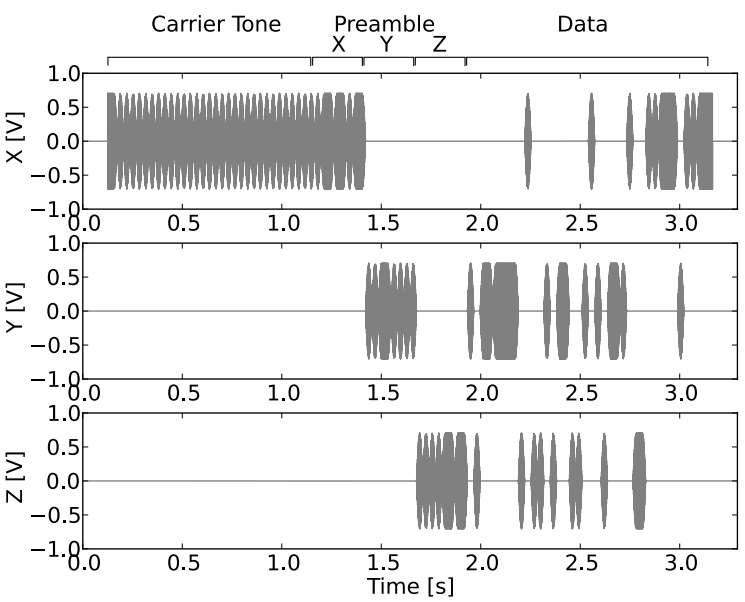

(a)

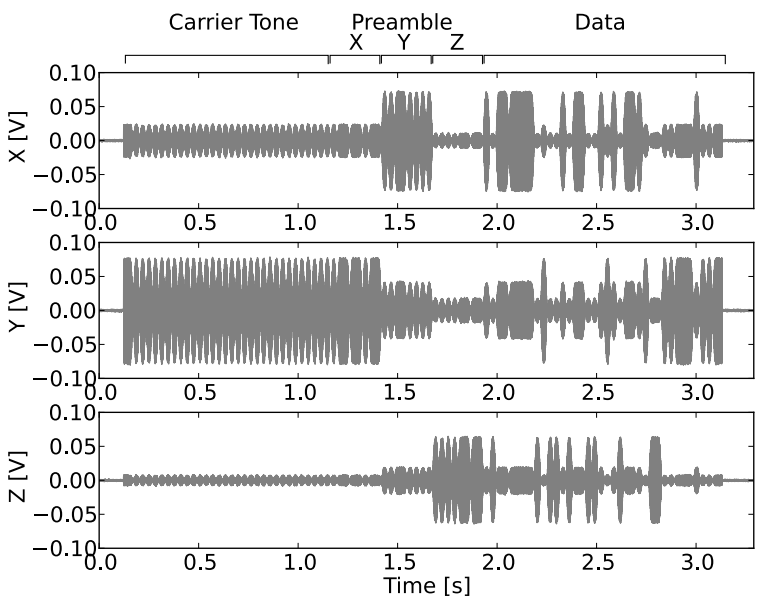

(b)

Figure 9: Triaxial transmitter and receiver waveforms. (a) shows the signal transmitted with a bitrate of $32 \mathrm{bps}$ and a carrier frequency of $2 \mathrm{kHz}$. Note how only one channel is active at each point in time and how the raised cosine filter smooths the envelope. (b) shows the received signal. Note how the signal received is a linear combination of the transmitted signal, with amplitude dependent on the active transmitter axis.

During this multichannel preamble, the receiver estimates the channel transfer function, which it uses to construct the constellation for symbol decoding. Finally, the data itself is sent.

\section{Range and Link Characterization}

In terms of maximum communication range, in an environment free from mains interference, the maximum achievable communication range was $35 \mathrm{~m}$. Mains interference has the effect of raising the noise floor and in an office environment the maximum achievable communication range was $23 \mathrm{~m}$. The variation in symbol error rate with distance is shown in Fig. 10, with both simulated and measured results in an office environment. Note the steepness of the error rate curve, due to the $60 \mathrm{~dB} /$ decade rolloff. A greater range would be possible using an optimized low noise amplifier and matched loop antenna.

\subsection{Network Results}

Broadcatching: Two transmitters were configured to send 8 byte messages to a single receiver in broadcatch mode. The spectrogram of the received broadcatch messages are shown in Fig. 11. The two input streams are frequency separated, with one having a carrier frequency of $2025 \mathrm{~Hz}$ and the other $2075 \mathrm{~Hz}$. These streams were successfully decoded, in parallel, thus demonstrating the merits of broadcatching. The $2025 \mathrm{~Hz}$ signal has been magnetic vector modulated (i.e. alternating on all three transmitting axes), whereas the $2075 \mathrm{~Hz}$ signal has been sent using a single axis transmitter using conventional BPSK. Note that they occupy identical bandwidths and although the message length is the same, the time taken for magnetic vector modulation is shorter.

\subsection{Combination}

The relative contribution of magnetic vector modulation is demonstrated, as these techniques are complementary and can either be used singly or together. As an example, the latency of conventional time-slotted network access is compared against the proposed broadcatching approach for

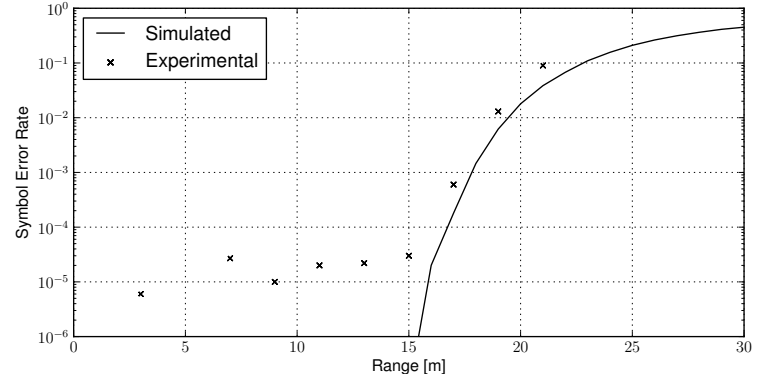

Figure 10: Variation in symbol error rate with distance.

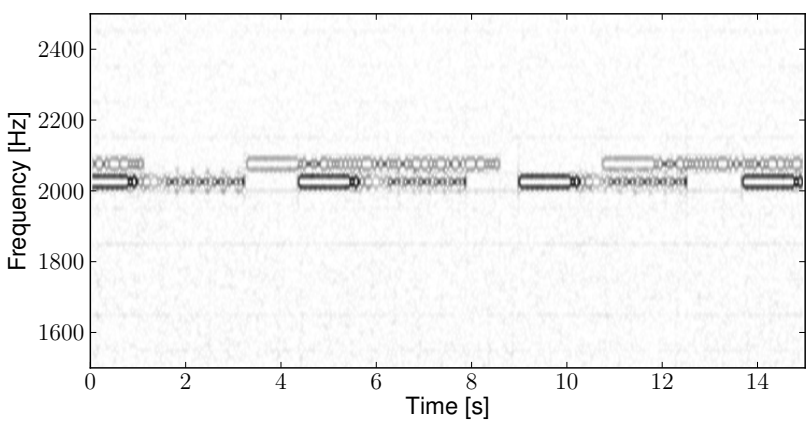

Figure 11: Spectrogram of the signal captured on the $\mathrm{x}$-axis coil showing broadcatching two transmitters sending an 8 byte message on $2025 \mathrm{~Hz}$ and $2075 \mathrm{~Hz}$. The bottom packet has been magnetic vector modulated and is hence quicker to send. 
a subtree with two children relaying data to a single parent, shown in Fig. 12 for various message lengths. In this experiment, two nodes were configured to send their data to a receiver. If both $\mathrm{A}$ and $\mathrm{B}$ occupy the same channel, they cannot send their data at the same time as it will collide at the receiver. This is shown as the solid line on the graph. If the broadcatching approach is used, A and B can transmit in the same time slot, effectively halving the latency, as reflected in the dash/dot line. It is interesting to note that in this two child example, magnetic vector modulation with two separate time slots can achieve lower latencies for larger messages than just using broadcatching alone. However, if three or more children are present, using broadcatching and the scalar transmission will always result in a lower latency. The largest performance gains are demonstrated using a combination of broadcatching and magnetic vector modulation, shown as the thick dotted line. For this two child example, the speedup relative to single channel, single axis communication is from $53 \mathrm{~s}$ to $11 \mathrm{~s}$, an improvement of over 4.5 times for a 100 byte message.

\section{RELATED WORK}

The use of multi-hop magneto-inductive networks was first introduced in [15], with the specific application of undersea communication. Underground sensor networks using a magneto-inductive communication channel were proposed in [2]. The use of magneto-inductive networks in a district heating system was presented in [14]. The use of a distributed magneto-inductive waveguide was suggested in [3], as a solution to the high path loss of the magnetic channel. Passive resonating coils are placed between the transmitter and receiver and act as a waveguide, reducing overall attenuation [16]. Magneto-inductive networks have also been proposed for monitoring leaks in pipelines [17]. The use of magneto-induction to localize animals underground was shown in [12], and more recently in [13], demonstrating a tracking accuracy of $0.45 \mathrm{~m}$ in $3-\mathrm{D}$. To date, the work in this area has considered the case of equipping each node with a single coil, which makes network operation highly dependent on network deployment. In the extreme case, if two coils are mutually perpendicular to each other, communication is impossible, as the receiver coil will not cut any magnetic flux lines. This is a serious drawback. However, through the use of three antennas, both on the transmitter and receiver, the presented system is omnidirectional and rotationally invariant. To the best of our knowledge, until now, no practical demonstration of low frequency communication in an underground sensor network using magneto-induction has been demonstrated.

It is important to note the distinction between conventional MIMO and magnetic vector modulation. MIMO relies on the fact that signals radiating from spatial distinct antennas travel over different path lengths through the diversity provided by a rich multipath environment, leading to delay spread [10]. In magnetic vector modulation, operation is within the near field and hence the channel response is purely real and as multipath does not occur. In addition, MIMO can be arbitrarily scaled according to the number of transmitter/receivers, subject to there being a rich scattering environment. Due to the extremely long wavelengths of the magnetic channel, it is not possible to arbitrarily scale it as closely spaced receiver loops will receive virtually identical signals.

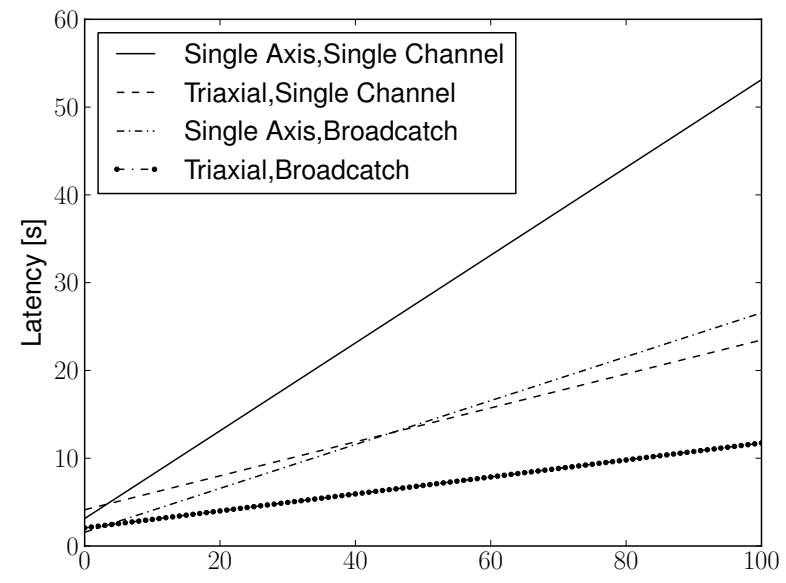

Figure 12: Latency for a node with two children, showing the relative merits of broadcatching and magnetic vector modulation with varying message lengths.

Multichannel trees have recently been suggested as a technique to improve data gathering performance and reduce network load [19]. This approach creates disjoint trees which are rooted at the same node but occupy distinct frequency bands. This is different to our work in that each receiver listens to a single input frequency. A recent work has considered the use two conventional radio receivers operating in different frequency bands [11], but has not considered the impact of this to reduce network latency.

\section{CONCLUSIONS AND FUTURE WORK}

In this work, we have considered a simple 6-ary method to modulate the magnetic vector. However, it is possible, through control of the relative amplitude and phase of currents in the three orthogonal coils to create arbitrary signal constellations, to increase bit rates or maximum communication range. Furthermore, using feedback from the receiver, the transmitter can alter the distribution of points in its constellation to avoid strong directional interference and optimize signal detection. A cognitive magnetic network could dynamically adapt to changing operational requirements or environmental conditions. Such a network is conceptually possible with our current software-defined prototype transceivers, and we suggest this as a future area of research.

In summary, in the event of a mining disaster, rapid and robust communication is essential in order to save lives. Through the use of magnetic vector transceivers, the system is made invariant to relative transceiver orientation and location, greatly simplifying deployment and making it robust to variations in position due to collapse or disturbance. The fundamental problem of the low frequency magnetic channel is the limited bitrate, mainly as a result of the extreme path loss of $60 \mathrm{~dB} /$ decade. We introduced magnetic vector modulation which exploits the triaxial antenna arrangement to improve the bit rate by over $250 \%$. When considering the network as a whole, further gains are made through the use of broadcatching, leading to over a four-fold decrease in system latency. Such techniques make the adoption of mag- 
netic transceiver nodes a reality. The unique characteristics of the magnetic channel are applicable to a number of different applications, such as indoor, underground or underwater networks where conventional $\mathrm{RF}$ is attenuated or subject to severe multipath.

\section{ACKNOWLEDGMENTS}

The authors would like to thank their shepherd Mani Srivastava and the anonymous reviewers for their helpful comments. This work was supported by the Oxford Martin School and EPSRC SUAAVE EP/F064217/1.

\section{REFERENCES}

[1] Census of fatal occupational injuries. Technical report, Burea of Labour and Statistics, USA, 2007.

[2] I. F. Akyildiz and E. P. Stuntebeck. Wireless underground sensor networks: Research challenges. Ad Hoc Networks, 4(6), 2006.

[3] I. F. Akyildiz, Z. Sun, and M. C. Vuran. Signal propagation techniques for wireless underground communication networks. Physical Communication, 2(3), 2009.

[4] A. Chehri, H. Mouftah, P. Fortier, and H. Aniss. Experimental Testing of IEEE801.15.4/ZigBee Sensor Networks in Confined Area. In Communication Networks and Services Research Conference (CNSR), 2010.

[5] J. N. de la Vergne. Hard Rock Miner's Handbook. McIntosh Engineering Limited, 2003.

[6] A. Farinelli, A. Rogers, A. Petcu, and N. R. Jennings. Decentralised coordination of low-power embedded devices using the max-sum algorithm. In $A A M A S$ '08, 2008.

[7] A. Farstad and R. Kehrman. Electromagnetic Location Systems for Metal/Non Metal Mines. BuMines OFR, 1979.

[8] R. Feynman. The Feynman lectures on physics. Addison Wesley Longman, 1970.

[9] R. A. Gates. Sago Mine Report ID No. 46-08791. Technical report, United States Department of Labor, 2007.

[10] D. Gesbert, M. Shafi, D. shan Shiu, P. Smith, and A. Naguib. From theory to practice: an overview of MIMO space-time coded wireless systems. Selected Areas in Communications, IEEE Journal on, 21(3):281 - 302, April 2003.
[11] B. Kusy, C. Richter, W. Hu, M. Afanasyev, R. Jurdak, M. Brunig, D. Abbott, C. Huynh, and D. Ostry. Radio diversity for reliable communication in WSNs. In Information Processing in Sensor Networks (IPSN), 2011.

[12] A. Markham, N. Trigoni, S. A. Ellwood, and D. W. Macdonald. Revealing the hidden lives of underground animals using magneto-inductive tracking. In Proceedings of the 8th ACM Conference on Embedded Networked Sensor Systems, 2010.

[13] A. Markham, N. Trigoni, D. Macdonald, and S. Ellwood. Underground localization in 3-D using magneto-inductive tracking. Sensors Journal, IEEE, PP(99):1, 2011.

[14] S. Meybodi, J. Nielsen, J. Bendtsen, and M. Dohler. Magneto-inductive underground communications in a district heating system. In Communications (ICC), 2011 IEEE International Conference on, pages $1-5$, June 2011.

[15] J. J. Sojdehei, P. N. Wrathall, and D. F. Dinn. Magneto-inductive (MI) communications. In Oceans 2001, MTS/IEEE Conference, Honolulu, USA, 2001.

[16] Z. Sun and I. Akyildiz. Magnetic induction communications for wireless underground sensor networks. Antennas and Propagation, IEEE Transactions on, 58(7):2426-2435, 2010.

[17] Z. Sun, P. Wang, M. C. Vuran, M. A. Al-Rodhaan, A. M. Al-Dhelaan, and I. F. Akyildiz. MISE-PIPE: Magnetic induction-based wireless sensor networks for underground pipeline monitoring. Ad Hoc Netw., 9:218-227, May 2011.

[18] M. C. Vuran and A. R. Silva. Communication Through Soil in Wireless Underground Sensor Networks:Theory and Practice. In Sensor Networks. Springer Berlin Heidelberg, 2009.

[19] Y. Wu, J. Stankovic, T. He, and S. Lin. Realistic and efficient multi-channel communications in wireless sensor networks. In INFOCOM 2008. The 27th Conference on Computer Communications. IEEE, pages 1193 -1201, April 2008.

[20] S. Yarkan, S. Guzelgoz, H. Arslan, and R. Murphy. Underground mine communications: A survey. Communications Surveys Tutorials, IEEE, 11(3):125 $-142,2009$.

[21] H. Yin and S. Alamouti. OFDMA: A Broadband Wireless Access Technology. In Sarnoff Symposium, 2006 IEEE, pages 1 -4, March 2006. 